\title{
Device-based antegrade dissection re-entry versus parallel wire techniques for the percutaneous revascularization of coronary chronic total occlusions
}

\author{
Qing Qin, Shufu Chang, Rende Xu, Lei Ge, Juying Qian, Jianying Ma, Junbo Ge
}

Department of Cardiology, Zhongshan Hospital, Fudan University,

Shanghai Institute of Cardiovascular Disease, Shanghai, China

\begin{abstract}
Background: Device-based antegrade dissection re-entry $(A D R)$ and parallel wire technique (PWT) are two important techniques in the antegrade approach in percutaneous coronary intervention (PCI) of chronic total occlusion (CTO). The study is aimed to compare the procedural and mid-term outcomes between device-based ADR using the CrossBoss/Stingray system and PWT in CTO PCI.

Methods: Data was retrospectively collected from consecutive patients who underwent CTO PCI using device-based ADR or PWT. CTO due to in-stent restenosis were excluded.

Results: A total of 273 patients were included in the study ( $n=55$ in device-based ADR group, $n=218$ in PWT group). Baseline characteristics were similar across groups except for higher prevalence of prior PCI and lower level of lipid profile in the ADR group. Moreover, although patients in the ADR group showed higher contrast volume $(441.6 \pm 162.4 \mathrm{~mL}$ vs. $361.5 \pm 142.1 \mathrm{~mL}, p<0.001)$, more intravascular ultrasound guidance (50.9\% vs. 22.9\%, $p<0.001$ ), more guidewires used $(4.6 \pm 1.4 v s$. $3.4 \pm 1.2, p<0.001)$ and higher troponin T level after PCI (0.167 vs. 0.087, $p=0.004)$, the technical success, procedural success and in-hospital complications were similar between the two groups. During a median follow-up of 1 year, the ADR group showed no difference in major adverse cardiac events (MACE, including all cause death, nonfatal myocardial infarction, and ischemia driven target vessel revascularization) ( $7.3 \%$ vs. $14.7 \%, p=0.150)$ as compared with the PWT group.

Conclusions: In the documented center, the use of device-based ADR for CTO PCI showed no difference in in-hospital complications and mid-term MACE as compared with PWT, despite higher procedure complexity in ADR group. (Cardiol J)
\end{abstract}

Key words: chronic total occlusion, antegrade dissection re-entry, parallel wire technique, Stingray, CrossBoss

\section{Introduction}

In the past decade, the technology related to chronic total occlusion (CTO) intervention has rapidly developed. Several significant breakthroughs in CTO wire technology and devices have resulted in an increasing antegrade success rate and a reduction of the retrograde approach [1]. During the antegrade approach, if the wire goes into the subintimal space, there are three commonly

Address for correspondence: Jianying $\mathrm{Ma}, \mathrm{MD}, \mathrm{PhD}$, and Junbo Ge, MD, PhD, Department of Cardiology, Zhongshan Hospital, Fudan University, Shanghai Institute of Cardiovascular Disease, NO 180, Fenglin Road, Shanghai, 200032 China, tel: +862164041990-5112 and +862164041990-5160, fax: +862164223006 , e-mail: ma.jianying@zs-hospital.sh.cn and ge.junbo@zs-hospital.sh.cn

Received: 11.06.2021 Accepted: 31.01.2022 Early publication date: 18.02.2022

This article is available in open access under Creative Common Attribution-Non-Commercial-No Derivatives 4.0 International (CC BY-NC-ND 4.0) license, allowing to download articles and share them with others as long as they credit the authors and the publisher, but without permission to change them in any way or use them commercially. 
used strategies, namely redirection, parallel wire technique (PWT) and device-based antegrade dissection re-entry (ADR) [1]. The CrossBoss and Stingray system (Boston Scientific, Marlborough, MA, USA) have been developed as a combination of devices that can create a controlled antegrade dissection plane to facilitate targeted distal vessel re-entry and have become the recommended mode of ADR in lesions which is characterized by a relatively disease-free re-entry zone, close proximity of the antegrade wire to the distal true lumen, and the absence of severe calcification in the re-entry zone [2]. According to available literature, the parallel wiring and ADR using Stingray offer better chances of success when compared with redirection [1]. Moreover, a meta-analysis published recently demonstrated comparable long-term clinical outcomes between limited dissection re-entry (including device-based ADR and reverse controlled antegrade and retrograde tracking [reverse CART]) and the wire escalation technique [3]. However, no comparison between PWT and device-based ADR has been made. The present study is aimed to compare the in-hospital and mid-term outcomes of device-based ADR vs. PWT for the percutaneous revascularization of coronary CTOs.

\section{Methods}

\section{Patient population}

Data was retrospectively collected from consecutive patients who underwent CTO percutaneous coronary intervention (PCI) by high volume operators ( $>75$ CTO PCI cases per year) [4], using device-based ADR or PWT in Zhongshan Hospital, Shanghai, China, between March 2015 and September 2019. Patients with CTO due to in-stent restenosis were excluded from the study. The study was approved by the institutional review board and all patients signed a general informed consent form.

\section{Study definitions and endpoints}

A CTO is defined as an occlusion with typical appearance (Thrombolysis in Myocardial Infarction [TIMI] grade 0 flow through the lesion with angiographically visible mature collaterals and the absence of thrombus or staining at the proximal cap) and definitive corroborating evidence of occlusion duration $\geq 3$ months according to CTO Academic Research Consortium (CTO-ARC) consensus recommendations [5]. The J-CTO score was calculated for all lesions [6]. Device-based ADR techniques were represented by using CrossBoss/Stingray system (Boston Scientific, USA). Patients were included in the device-based ADR group if they were ever treated with either device. Technical success is defined as achievement of TIMI grade 2 or greater antegrade flow in all $\geq 2.5$-mm distal branches with $<30 \%$ residual stenosis of the target CTO lesion [5]. Procedural success was defined as technical success in the absence of in-hospital adverse events, including all-cause death, nonfatal myocardial infarction (MI), ischemia driven target vessel revascularization (TVR) with PCI or coronary artery bypass grafting (CABG). In-hospital complications included loss of side branches, coronary perforation, cardiac tamponade, nonfatal MI, ischemia driven TVR, and all cause death. Major adverse cardiac events (MACEs) on follow-up were defined as the composite of all cause death, nonfatal $\mathrm{MI}$, and ischemia driven TVR.

\section{Procedure}

All patients were treated with acetylsalicylic acid (ASA) and a loading dose of a thienopyridine before the procedure. During the procedure, unfractionated heparin was administered intravenously at $100 \mathrm{IU} / \mathrm{kg}$ followed by further heparin as necessary to achieve a target activated clotting time of 250-350 s. Glycoprotein (GP) IIb/IIIa inhibitors were administered at operator's discretion [7]. After careful evaluation of the diagnostic coronary angiogram, the operator chose the primary approach (antegrade or retrograde). In patients with technical success, dual antiplatelets therapy was started after the procedure with ASA and thienopyridine and was maintained for at least 12 months.

\section{Data collection}

Demographic, procedural, and outcome data were obtained from review of the catheterization laboratory database and medical chart review. Clinical follow-up data were collected through outpatient visits, telephone interviews and medical chart reviews.

\section{Statistical analysis}

Continuous variables are presented as mean \pm standard deviation or the median with interquartile range and were compared by the Student t-test or the Mann-Whitney U test, respectively. Categorical variables are presented as counts and percentages and were compared by the $\chi^{2}$ test (or the Fisher exact test when appropriate). Kaplan-Meier curves of survival free from MACE were plotted in ADR and PWT group respectively and compared using 
Table 1. Baseline clinical characteristics.

\begin{tabular}{|c|c|c|c|}
\hline & \multicolumn{2}{|c|}{ All patients } & \multirow[t]{2}{*}{$\mathbf{P}$} \\
\hline & $\operatorname{ADR}(n=55)$ & PWT (n = 218) & \\
\hline Age [years] & $63.5 \pm 10.7$ & $61.9 \pm 11.7$ & 0.348 \\
\hline Male gender & $52(94.5 \%)$ & $185(84.9 \%)$ & 0.073 \\
\hline Hypertension & $33(60.0 \%)$ & $140(64.2 \%)$ & 0.639 \\
\hline Diabetes & $16(29.1 \%)$ & $59(27.1 \%)$ & 0.739 \\
\hline Insulin & $5(9.1 \%)$ & $22(10.1 \%)$ & 1.000 \\
\hline Dyslipidemia & $4(7.3 \%)$ & $19(8.7 \%)$ & 0.490 \\
\hline Smoking & $25(45.5 \%)$ & $105(48.2 \%)$ & 0.764 \\
\hline Drink & $5(9.1 \%)$ & $15(6.9 \%)$ & 0.375 \\
\hline Previous MI & $18(32.7 \%)$ & $47(21.6 \%)$ & 0.110 \\
\hline Previous $\mathrm{PCl}$ & $36(65.5 \%)$ & $96(44.0 \%)$ & 0.005 \\
\hline Previous CABG & $4(7.3 \%)$ & $8(3.7 \%)$ & 0.268 \\
\hline Diagnosis: & & & 0.234 \\
\hline Stable angina & $19(34.5 \%)$ & $83(38.1 \%)$ & \\
\hline Unstable angina & $19(34.5 \%)$ & $85(39.0 \%)$ & \\
\hline STEMI & $0(0 \%)$ & $2(0.9 \%)$ & \\
\hline NSTEMI & $3(5.5 \%)$ & $20(9.2 \%)$ & \\
\hline Silent ischemia & $14(25.5 \%)$ & $28(12.8 \%)$ & \\
\hline \multicolumn{4}{|l|}{ Lab test: } \\
\hline $\mathrm{TC}[\mathrm{mmol} / \mathrm{L}]$ & $3.3 \pm 0.8$ & $3.8 \pm 1.2$ & 0.002 \\
\hline $\mathrm{TG}[\mathrm{mmol} / \mathrm{L}]$ & $1.6 \pm 0.7$ & $2.0 \pm 1.4$ & 0.001 \\
\hline LDL-C [mmol/L] & $1.6 \pm 0.7$ & $2.0 \pm 1.1$ & 0.005 \\
\hline HDL-C [mmol/L] & $1.0 \pm 0.2$ & $1.0 \pm 0.3$ & 0.228 \\
\hline eGFR $\left[\mathrm{mL} / \mathrm{min} / 1.73 \mathrm{~m}^{2}\right]$ & $82.2 \pm 19.1$ & $87.8 \pm 21.4$ & 0.079 \\
\hline $\mathrm{HbA} 1 \mathrm{c}[\%]$ & $6.2 \pm 1.0$ & $6.4 \pm 1.3$ & 0.275 \\
\hline LVEF [\%] & $59.1 \pm 11.8$ & $58.9 \pm 9.8$ & 0.921 \\
\hline
\end{tabular}

ADR - antegrade dissection re-entry; PWT — parallel wire technique; $\mathrm{MI}$ - myocardial infarction; $\mathrm{PCl}$ - percutaneous coronary intervention; CABG - coronary artery bypass grafting; STEMI — ST-segment elevation myocardial infarction; NSTEMI - non-ST-segment elevation myocardial infarction; TC - total cholesterol; TG - triglyceride; LDL-C - low-density lipoprotein cholesterol; HDL-C - high-density lipoprotein cholesterol; eGFR - estimated glomerular filtration rate; HbA1c - hemoglobin A1c; LVEF — left ventricular ejection fraction

the log-rank test. Logistic regression with Enter method was used for multivariate analysis of independent correlates of technical success. The candidate variables for the model were selected based on significant univariate analysis and clinically relevance. All analyzes were performed using SPSS, Version 20.0 (IBM Corporation, Armonk, NY, USA), and a p value of $<0.05$ was considered statistically significant.

\section{Results}

\section{Baseline characteristics between ADR and PWT groups}

From March 2015 to September 2019, 273 CTO patients managed by device-based ADR $(n=55)$ or PWT ( $\mathrm{n}=218$ ) were consecutively enrolled in the present study. In the ADR group, CrossBoss catheter was used in 6 patients, Stingray system was used in 41 patients and both devices were used in 8 patients. The baseline clinical characteristics are listed in Table 1. Compared with the PWT group, patients in the ADR group had a higher prevalence of prior PCI (65.5\% vs. 44.0, $\mathrm{p}=0.005$ ), whereas lower level of lipid profile including total cholesterol (TC), low-density lipoprotein cholesterol (LDL-C), and triglycerides (TG).

Angiographic and procedural characteristics Angiographic and procedural data are presented in Table 2 . The angiographic characteristics were comparable between the two groups except 
Table 2. Angiographic and procedural characteristics.

\begin{tabular}{|c|c|c|c|}
\hline & \multicolumn{2}{|c|}{ All patients } & \multirow[t]{2}{*}{$\mathbf{P}$} \\
\hline & ADR $(n=55)$ & PWT $(n=218)$ & \\
\hline Number of diseased vessels & $2.2 \pm 0.7$ & $2.2 \pm 0.8$ & 0.948 \\
\hline Target CTO vessel: & & & 0.022 \\
\hline LAD & $25(45.5 \%)$ & $79(36.2 \%)$ & \\
\hline LCX & $1(1.8 \%)$ & $30(13.8 \%)$ & \\
\hline RCA & $29(52.7 \%)$ & $109(50.0 \%)$ & \\
\hline Blunt Stump & $30(54.5 \%)$ & $118(54.1 \%)$ & 1.000 \\
\hline Moderate or severe calcifications & $4(7.3 \%)$ & $35(16.1 \%)$ & 0.130 \\
\hline$>45$ degree bending & $15(27.3 \%)$ & $58(26.6 \%)$ & 1.000 \\
\hline Lesion length $>20 \mathrm{~mm}$ & $37(67.3 \%)$ & $125(57.3 \%)$ & 0.219 \\
\hline Retry & $22(40.0 \%)$ & $61(28.0 \%)$ & 0.101 \\
\hline J-CTO score & $2.0 \pm 0.9$ & $1.8 \pm 1.1$ & 0.318 \\
\hline Absence of interventional collaterals & $14(25.5 \%)$ & $76(34.9 \%)$ & 0.203 \\
\hline IVUS-guided procedure & $28(50.9 \%)$ & $50(22.9 \%)$ & $<0.001$ \\
\hline Number of CTO guidewires used & $4.6 \pm 1.4$ & $3.4 \pm 1.2$ & $<0.001$ \\
\hline \multicolumn{4}{|c|}{ Microcatheter used in antegrade approach: } \\
\hline Finecross & $14(25.5 \%)$ & $74(33.9 \%)$ & 0.229 \\
\hline Corsair & $36(65.5 \%)$ & $122(56.0 \%)$ & 0.203 \\
\hline Crusade & $20(36.4 \%)$ & $113(51.8 \%)$ & 0.040 \\
\hline Other & $0(0 \%)$ & $14(6.5 \%)$ & - \\
\hline \multicolumn{4}{|l|}{ Primary approach: } \\
\hline Antegrade & $50(90.9 \%)$ & $211(96.8 \%)$ & 0.070 \\
\hline ADR & $35(63.6 \%)$ & & \\
\hline PWT & $15(27.3 \%)$ & & \\
\hline Retrograde & $5(9.1 \%)$ & $7(3.2 \%)$ & 0.070 \\
\hline Recanalization technique: & & & - \\
\hline ADR & $32(58.2 \%)$ & $0(0 \%)$ & - \\
\hline PWT & $1(1.8 \%)$ & $146(67.0 \%)$ & - \\
\hline Single wire & $1(1.8 \%)$ & $1(0.5 \%)$ & - \\
\hline Retrograde & $7(12.7 \%)$ & $27(12.4 \%)$ & - \\
\hline Average stent diameter $[\mathrm{mm}]$ & $3.0 \pm 0.3$ & $3.0 \pm 0.3$ & 0.302 \\
\hline Total stent length [mm] & $86.4 \pm 23.4$ & $77.0 \pm 28.8$ & 0.054 \\
\hline Contrast volume [mL] & $441.6 \pm 162.4$ & $361.5 \pm 142.1$ & $<0.001$ \\
\hline Technical success & $41(74.5 \%)$ & $174(79.8 \%)$ & 0.460 \\
\hline Procedural success & $41(74.5 \%)$ & $172(78.9 \%)$ & 0.486 \\
\hline In-hospital days & $5.0 \pm 2.7$ & $5.2 \pm 3.6$ & 0.777 \\
\hline \multicolumn{4}{|l|}{ Pre-PCI cardiac enzyme: } \\
\hline Troponin T [ng/mL] & $0.016(0.009-0.025)$ & $0.011(0.008-0.023)$ & 0.222 \\
\hline CK-MB [U/L] & $15.3 \pm 6.2$ & $15.0 \pm 8.4$ & 0.824 \\
\hline \multicolumn{4}{|l|}{ Post-PCl cardiac enzyme: } \\
\hline Troponin T [ng/mL] & $0.167(0.073-0.311)$ & $0.087(0.036-0.234)$ & 0.004 \\
\hline CK-MB [U/L] & $24.2 \pm 13.4$ & $22.6 \pm 18.5$ & 0.574 \\
\hline
\end{tabular}

ADR - antegrade dissection re-entry; PWT — parallel wire technique; CTO — chronic total occlusion; LAD — left anterior descending coronary artery; LCX — left circumflex coronary artery; RCA — right coronary artery; IVUS — intravascular ultrasound; PCI — percutaneous coronary intervention; $\mathrm{CM}-\mathrm{MB}$ - creatine kinase-MB 
Table 3. In-hospital complications.

\begin{tabular}{lccc}
\hline & \multicolumn{2}{c}{ All patients } & P \\
\cline { 2 - 3 } & ADR $(\mathbf{n}=55)$ & PWT $(\mathbf{n}=218)$ \\
\hline Loss of side branches & $11(20 \%)$ & $26(11.9 \%)$ & 0.118 \\
Perforation & $4(7.3 \%)$ & $4(1.8 \%)$ & 0.055 \\
Cardiac tamponade & $2(3.6 \%)$ & $1(0.5 \%)$ & 0.104 \\
Myocardial infarction & $0(0 \%)$ & $1(0.5 \%)$ & - \\
Ischemia-driven TVR & $0(0 \%)$ & $0(0 \%)$ & - \\
Death & $0(0 \%)$ & $1(0.5 \%)$ & - \\
\hline
\end{tabular}

ADR — antegrade dissection re-entry; PWT — parallel wire technique; TVR — target vessel revascularization

that more left circumflex coronary artery (LCX) CTOs were treated in PWT group when compared with ADR group. There was no difference in technical success $(74.5 \%$ in ADR vs. $79.8 \%$ in PWT, $\mathrm{p}>0.05)$ and procedural success $(70.9 \%$ in ADR vs. $78.4 \%$ in PWT, $p>0.05$ ) between the two groups. Patients in ADR group showed higher percentage of intravascular ultrasound (IVUS) guidance $(50.9 \%$ vs. $22.9 \%, \mathrm{p}<0.001)$, more CTO guidewires $(4.6 \pm 1.4$ vs. $3.4 \pm 1.2, \mathrm{p}<0.001)$ and contrast volume $(441.6 \pm 162.4 \mathrm{~mL}$ vs. $361.5 \pm$ $\pm 142.1 \mathrm{~mL}, \mathrm{p}<0.001)$ used in the procedure, higher troponin $\mathrm{T}$ level after the procedure $(0.167$ vs. $0.087 \mathrm{ng} / \mathrm{mL}, \mathrm{p}=0.004$ ), and lower percentage of Crusade microcatheter (36.4\% vs. $51.8 \%$, $\mathrm{p}=0.04)$ used in the procedure.

\section{In-hospital complications}

In-hospital complications are shown in Table 3. In PWT group, 2 cases of coronary perforation were collateral channel perforation after the retrograde technique and were managed by coil embolization, while the other 2 cases were main vessel perforation (mid left anterior descending coronary artery [LAD] and distal right coronary artery [RCA] which were managed by balloon inflation). In ADR group, 3 cases of coronary perforation were collateral channel perforation after the retrograde technique and were managed by coil embolization, while 1 case was main vessel perforation in CrossBoss treated patients. Although no coronary perforation was observed at final angiogram, 2 patients using Stingray balloon in ADR group and 1 patient in PWT group suffered from cardiac tamponade several hours after the procedure and pericardiocentesis was performed. One case of MI occurred during the procedure in PWT group, which was caused by acute stent thrombosis in distal RCA stent during the retrograde approach to recanalize
LAD CTO lesion. One patient died in PWT group due to cerebral hemorrhage as confirmed by computed tomography scan after the procedure. There was a trend of more coronary perforation in ADR group $(\mathrm{p}=0.055)$.

\section{Clinical outcomes on follow-up}

Median follow-up was 365 days (interquartile range 249-527 days) in ADR group and 370 days (interquartile range 171-586 days) in PWT group. There were no significant differences in the rates of MACE between groups. In particular, the MACE rate was $7.3 \%$ in ADR group and $14.7 \%$ in PWT group (Table 4). Kaplan-Meier curves of MACE-free survival at 3 years of follow-up are shown in Figure 1 and there was no significant difference between the two groups $(p=0.150)$.

\section{Independent predictors of technical success}

In the multivariate logistic regression analysis, hypertension (odds ratio [OR]: 0.347, 95\% confidence interval [CI]: 0.169-0.711, $\mathrm{p}=0.004$ ), LDL-C (OR: 0.701, 95\% CI: 0.521-0.943, $\mathrm{p}=0.019$ ) and lesion length $>20 \mathrm{~mm}$ (OR: $0.345,95 \% \mathrm{CI}$ : $0.172-0.691, \mathrm{p}=0.003$ ) were identified as independent correlates of technical success in CTO patients (Table 5).

\section{Discussion}

According to available research, this study is the first to compare in-hospital and mid-term outcomes of device-based ADR vs. PWT for the PCI of coronary CTOs. Findings herein, suggested that there were no differences in the procedural and mid-term outcomes between these two strategies. Besides hypertension, LDL-C and lesion length $>20 \mathrm{~mm}$ were identified as independent correlates of technical success in CTO patients. 
Table 4. Mid-term follow-up results.

\begin{tabular}{lccc}
\hline & \multicolumn{2}{c}{ All patients } & \\
\cline { 2 - 3 } & ADR $(\mathbf{n}=\mathbf{5 5})$ & PWT $(\mathbf{n}=\mathbf{2 1 8})$ \\
\hline MI: & 0 & 3 & - \\
STEMI & 0 & 0 & - \\
NSTEMI & 0 & 3 & - \\
Death: & 0 & 2 & - \\
Cardiac & 0 & 1 & - \\
Non-cardiac & 0 & 1 & - \\
TVR: & 4 & 29 & - \\
PCI & 3 & 22 & - \\
CABG & 1 & 7 & 0.150 \\
MACE & $4(7.3 \%)$ & $32(14.7 \%)$ & 0.802 \\
Follow up days & $365(249-527)$ & $370(171-586)$ & \\
\hline
\end{tabular}

ADR - antegrade dissection re-entry; PWT — parallel wire technique; MI — myocardial infarction; STEMI — ST-segment elevation myocardial infarction; NSTEMI — non-ST segment elevation myocardial infarction; TVR — target vessel revascularization; PCI — percutaneous coronary intervention; CABG — coronary artery bypass grafting; MACE - major adverse cardiac event

Table 5. Predictors of procedure success in chronic total occlusion lesion

\begin{tabular}{lcccccc}
\hline & $\beta$ coefficient & Wald & P & OR & \multicolumn{2}{c}{$95 \%$ Cl of OR } \\
\hline Hypertension & -1.058 & 8.364 & 0.004 & 0.347 & 0.169 & 0.711 \\
LDL-C & -0.356 & 5.523 & 0.019 & 0.701 & 0.521 & 0.943 \\
Lesion length $>20 \mathrm{~mm}$ & -1.064 & 9.023 & 0.003 & 0.345 & 0.172 & 0.691 \\
Absence of interventional collaterals & 0.518 & 2.319 & 0.128 & 1.679 & 0.862 & 3.27 \\
Antegrade dissection re-entry & -0.408 & 1.17 & 0.279 & 0.665 & 0.318 & 1.393 \\
\hline
\end{tabular}

$\mathrm{Cl}$ - confidence interval; LDL-C - low-density lipoprotein cholesterol; OR - odds ratio

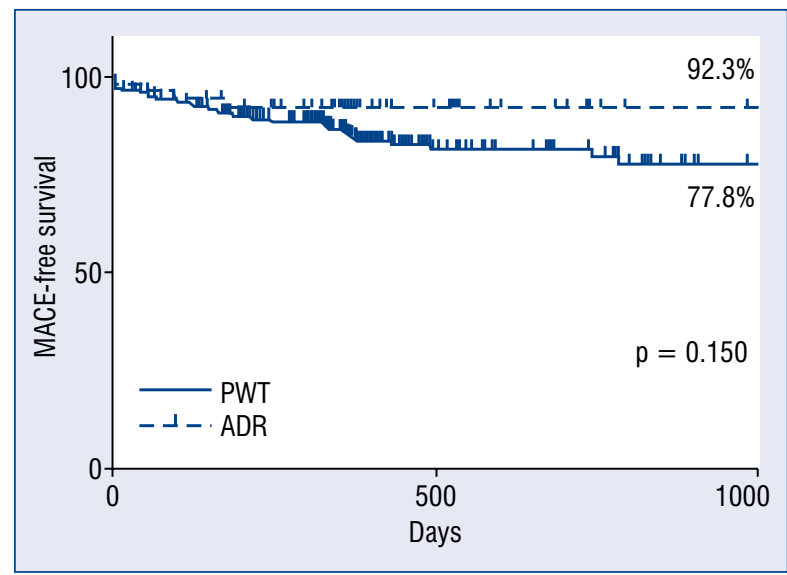

Figure 1. The Kaplan-Meier curves of major adverse cardiac event (MACE)-free survival in chronic total occlusion patients treated with antegrade dissection reentry (ADR) and parallel wire technique (PWT) strategy.
Antegrade dissection re-entry for CTO PCI was first described as the subintimal tracking and re-entry (STAR) technique 15 years ago [8]. This involves pushing a folded or 'Knuckled' (usually polymer jacketed) guidewire in an antegrade direction through the subintimal space until it re-enters the distal true lumen. Although STAR enabled successful recanalization of long, tortuous and ambiguous occlusion, high rates of restenosis (54\%) occurred 5 months after STAR [8]. Contrast-guided STAR [9], Mini-STAR [10], and limited antegrade subintimal tracking (LAST) [11] are derived from STAR and are included in wire-based ADR. Even contrast-guided STAR and Mini-STAR showed high rates of restenosis (25-54\%) on 2-year follow-up $[9,10]$. The 1-year MACE (death, MI and TVR) rate of LAST is $17.5 \%$, which is comparable to STAR (15.4\%) as reported [11]. The high restenosis rate 
in wire-based ADR may be attributed to poor distal runoff due to long dissections and loss of side branches [11, 12].

To minimize vessel trauma during dissection, device-based ADR was introduced into contemporary ADR practice, in which subintimal lesion crossing can be performed with controlled dissection using the CrossBoss microcatheter and reentry can be achieved with Stingray system. The technical success rate of the CrossBoss/Stingray system in CTO lesion was $67-89 \%$ as reported [11, $13,14]$, which was higher than that of STAR (59\%) in wire-based ADR [11] and was similar to other CTO crossing strategies (76.2\%) [14]. Moreover, device-based ADR also showed lower MACE (cardiac death, target-vessel MI, and ischemia driven TVR) rates (4.3\%) when compared with wire-based ADR (16.9\%) during 24-month followup. In the present study, technical success was found in device-based ADR at $74.5 \%$ and MACE (all-cause death, nonfatal MI, and ischemia-driven TVR) rate was 7.3\% during 1-year follow-up, which was consistent with the previous studies. Although 1 study reported high MACE (all-cause mortality, MI, unplanned repeat coronary revascularization, and stroke) rate (40.3\%) in device-based ADR group during 1.8-year follow-up, this may be attributed to high rates of prior CABG and diffuse coronary artery disease in that study [14].

Parallel wire technique is another commonly used technique in the antegrade approach besides ADR. In this technique, the initial guidewire is left in the CTO body as a marker and to obstruct the false channel, and then a second wire is inserted along it to cross the CTO lesion. Although PWT is widely used in CTO PCI, minimal published data exist on this technique and its outcomes. In the current study, a technical success rate of $79.8 \%$ was demonstrated and MACE rate of $14.7 \%$ in PWT group, which is similar to that of device-based ADR. Furthermore, using ADR or PWT showed no correlation with technical success in multivariate logistic regression. Lesion length $>20 \mathrm{~mm}$ was identified as independent correlates of technical success in this study, which is consistent with the RECHARGE and J-CTO score system [6, 15].

Furthermore, dual lumen microcatheter Crusade (KANEKA) was used in $51.8 \%$ PWT cases. Dual lumen microcatheter was designed for managing bifurcation lesions, however, nowadays it is used in PWT in CTO lesions by leaving the first wire in the monorail lumen while manipulating the second wire in the over the wire (OTW) lumen to enter the true lumen. The first wire in the monorail lumen can fix and stabilize the microcatheter, make strong backup and improve the operability of the second guidewire [16]. Whether dual lumen microcatheter can improve the technical success rate in PWT is still unknown, in our center however, there is growing use of dual lumen microcatheter in PWT.

Both PWT and device-based ADR may create substantial subintimal space expansion, leading to side branch loss and myocardial enzyme elevation after PCI. In the current study, although no statistically significant difference was detected in side branch loss between ADR (20\%) and PWT (11.9\%) group after PCI ( $p>0.05)$, the troponin T level after PCI was substantially higher in ADR group than in PWT group $(\mathrm{p}=0.004)$, indicating more severe injury to the vessel in ADR group. J-CTO score was identical between ADR and PWT groups, but patients in ADR group had more prior PCI attempts, higher contrast volume, more IVUS guidance, and more guidewires used, which showed a more complex PCI procedure in ADR group as reported [14]. Perforation was a concern in device-base ADR, and was reported in $1.6-2.6 \%$ of cases in the previous studies $[11,14]$. In the present study, perforation occurred in 4 cases in ADR group, of which only one was caused by CrossBoss. There are also 4 cases of perforation in PWT group, which indicates no higher perforation risk in $\mathrm{ADR}$ group.

The hybrid CTO PCI strategy places emphasis on procedural efficiency, and recommends that changes of strategy should occur very early, and often cycle rapidly, to maximize the likelihood of early successful crossing [17]. As PWT and ADR increased peri-wire space expansion and risk of hematoma formation, it is proper to switch between ADR and PWT early if either technique has not resulted in any significant progress before peri-wire space expansion [1]. Only 1 case in the current study was recanalized by PWT after failure of ADR, while 9 cases succeeded by ADR after PWT failure. This is probably due to the larger peri-wire space induced by ADR compared with PWT in this study, which makes the manipulation of the guidewire difficult in PWT, implying that switching from ADR to PWT at an early stage of the procedure may be effective.

\section{Limitations of the study}

First, this is an observational study, and thus may have bias related to the study design. Therefore, prospective, randomized clinical trials are needed to assess the outcomes of CTO lesions treated with device-based ADR compared with 
PWT. Second, the sample size in ADR group is relatively small and there may be type-II error when analyzing differences of variables. Third, this is a single center study with high-volume CTO operators involved, the results may not be generalized due to differences in CTO PCI experience. Forth, CrossBoss was used in 14 (25.5\%) patients in ADR group in this study, which is relatively low as compared with data reported previously ranging from $63 \%$ to $85 \%[14,18]$. The preference of device in ADR limited the generalization of the results. Fifth, as improvements in symptoms and quality of life were not evaluated in the study, some information might have been missed in the mid-term follow-up.

\section{Conclusions}

In the present center, use of device-base ADR for CTO PCI showed no difference in technical success, in-hospital complications and mid-term MACE as compared with PWT, despite higher procedural complexity in ADR group.

\section{Funding}

This work was supported by National Key R\&D Program of China (No. 2019YFC0120200) and the National Natural Science Foundation of China (No. 81570315) to Q.Q., and the Clinical Science and Technology Innovation Project of Shanghai Shenkang Hospital Development Center (SHDC12018X05) to M.JY.

\section{Conflict of interest: None declared}

\section{References}

1. Wu EB, Tsuchikane E, Lo S, et al. Chronic total occlusion wiring: a state-of-the-art guide from the asia pacific chronic total occlusion club. Heart Lung Circ. 2019; 28(10): 1490-1500, doi: 10.1016/j. hlc.2019.04.004, indexed in Pubmed: 31128985.

2. Walsh SJ, Cosgrove C, Spratt JC, et al. A technical focus on antegrade dissection and re-entry for coronary chronic total occlusions: a practice update for 2019. Korean Circ J. 2019; 49(7): 559—567, doi: 10.4070/ kcj.2019.0160, indexed in Pubmed: 31243929.

3. Zhao Y, Peng H, Li X, et al. The impact of dissection and re-entry versus wire escalation techniques on long-term clinical outcomes in patients with chronic total occlusion lesions following percutaneous coronary intervention: An updated meta-analysis. Cardiol J. 2021; 28(3): 369-383, doi: 10.5603/CJ.a2020.0026, indexed in Pubmed: 32104900.

4. Lee CK, Chen YH, Lin MS, et al. Retrograde approach is as effective and safe as antegrade approach in contemporary percutaneous coronary intervention for chronic total occlusion: a taiwan single-center registry study. Acta Cardiol Sin. 2017; 33(1): 20-27, doi: 10.6515/acs20160131a, indexed in Pubmed: 28115803.

5. Ybarra LF, Rinfret S, Brilakis ES, et al. Definitions and clinical trial design principles for coronary artery chronic total occlusion therapies:
CTO-ARC consensus recommendations. Circulation. 2021; 143(5): 479-500, doi: 10.1161/CIRCULATIONAHA.120.046754, indexed in Pubmed: 33523728.

6. Morino Y, Abe M, Morimoto T, et al. J-CTO Registry Investigators. Predicting successful guidewire crossing through chronic total occlusion of native coronary lesions within 30 minutes: the J-CTO (Multicenter CTO Registry in Japan) score as a difficulty grading and time assessment tool. JACC Cardiovasc Interv. 2011; 4(2): 213-221, doi: 10.1016/j.jcin.2010.09.024, indexed in Pubmed: 21349461.

7. Qin Q, Chang S, Xu R, et al. Short and long-term outcomes of coronary perforation managed by coil embolization: A single-center experience. Int J Cardiol. 2020; 298: 18-21, doi: 10.1016/j. ijcard.2019.07.091, indexed in Pubmed: 31402155.

8. Colombo A, Mikhail GW, Michev I, et al. Treating chronic total occlusions using subintimal tracking and reentry: the STAR technique. Catheter Cardiovasc Interv. 2005; 64(4): 407-412, doi: 10.1002/ ccd.20307, indexed in Pubmed: 15789384.

9. Godino C, Latib A, Economou FI, et al. Coronary chronic total occlusions: mid-term comparison of clinical outcome following the use of the guided-STAR technique and conventional anterograde approaches. Catheter Cardiovasc Interv. 2012; 79(1): 20-27, doi: 10.1002/ ccd.23058, indexed in Pubmed: 21805559.

10. Galassi AR, Boukhris M, Tomasello SD, et al. Long-term clinical and angiographic outcomes of the mini-STAR technique as a bailout strategy for percutaneous coronary intervention of chronic total occlusion. Can J Cardiol. 2014; 30(11): 1400-1406, doi: 10.1016/j. cjca.2014.07.016, indexed in Pubmed: 25442438.

11. Azzalini L, Dautov R, Brilakis ES, et al. Procedural and longer-term outcomes of wire- versus device-based antegrade dissection and reentry techniques for the percutaneous revascularization of coronary chronic total occlusions. Int J Cardiol. 2017; 231: 78-83, doi: 10.1016/j. ijcard.2016.11.273, indexed in Pubmed: 27887799.

12. Carlino M, Figini F, Ruparelia N, et al. Predictors of restenosis following contemporary subintimal tracking and reentry technique: The importance of final TIMI flow grade. Catheter Cardiovasc Interv. 2016; 87(5): 884-892, doi: 10.1002/ccd.26103, indexed in Pubmed: 26308750.

13. Whitlow PL, Burke MN, Lombardi WL, et al. Use of a novel crossing and re-entry system in coronary chronic total occlusions that have failed standard crossing techniques: results of the FAST-CTOs (Facilitated Antegrade Steering Technique in Chronic Total Occlusions) trial. JACC Cardiovasc Interv. 2012; 5(4): 393-401, doi: 10.1016/j. jcin.2012.01.014, indexed in Pubmed: 22516395.

14. Mogabgab O, Patel VG, Michael TT, et al. Long-term outcomes with use of the CrossBoss and stingray coronary CTO crossing and reentry devices. J Invasive Cardiol. 2013; 25(11): 579-585, indexed in Pubmed: 24184892.

15. Maeremans J, Spratt JC, Knaapen P, et al. Towards a contemporary, comprehensive scoring system for determining technical outcomes of hybrid percutaneous chronic total occlusion treatment: The RECHARGE score. Catheter Cardiovasc Interv. 2018; 91(2): 192-202, doi: 10.1002/ccd.27092, indexed in Pubmed: 28471074.

16. Tanabe G, Oikawa Y, Yajima J, et al. Retrograde parallel wire technique using a dual lumen catheter can be useful for percutaneous coronary intervention with chronic total occlusion. J Cardiol Cases. 2018; 17(1): 25-28, doi: 10.1016/j.jccase.2017.08.014, indexed in Pubmed: 30279847.

17. Brilakis ES, Grantham JA, Rinfret S, et al. A percutaneous treatment algorithm for crossing coronary chronic total occlusions. JACC Cardiovasc Interv. 2012; 5(4): 367-379, doi: 10.1016/j.jcin.2012.02.006, indexed in Pubmed: 22516392.

18. Christopoulos G, Karmpaliotis D, Alaswad K, et al. Application and outcomes of a hybrid approach to chronic total occlusion percutaneous coronary intervention in a contemporary multicenter US registry. Int J Cardiol. 2015; 198: 222-228, doi: 10.1016/j.ijcard.2015.06.093, indexed in Pubmed: 26189193. 